\title{
POSTER
}

\section{Plaques d'ancrage orthodontiques : Techniques et indications actuelles}

\section{Afota $\mathrm{F}^{1}$, Olimpo $\mathrm{M}^{2}$, Maniere $\mathrm{A}^{3}$, Cochais $\mathrm{P}^{4}$, Savoldelli $\mathrm{C}^{5}$}

1 - Interne en Chirugie Orale, Service de Chirurgie Orale et Maxillo Faciale, CHU Nice*

2 - Interne en Orthopédie Dento Faciale, Service Odontologie, CHU Nice*

3 - PU-PH en Orthopédie Dento Faciale, Service Odontologie, CHU Nice ${ }^{\star \star}$

Doyen de l'UFR Odontologie, Université de Nice Sophia Antipolis

$4 \mathrm{MCU}-\mathrm{PH}$, Service Odontologie, CHU Nice ${ }^{\star *}$

$5 \mathrm{MCU}$ - PH, Service de Chirurgie Orale et Maxillo Faciale, CHU Nice*

*Institut Universitaire de la Face et du Cou, 31 Avenue de Valombrose 06000 NICE

** Hopital St Roch, 5 Rue Pierre Devoluy, 06000 NICE

L'ancrage osseux par mini-plaques en titane est en plein essor. Ces plaques offrent des possibilités de mobilisation orthodontique de grande amplitude sans effets parasites résiduels. Leurs indications étaient initialement réservées aux mécaniques « orthopédiques » en sollicitant les bases osseuses et se sont étendues aux mouvements dentaires d'ingression, de mésialisation ou de distalisation de masse. Ces plaques offrent à l'orthodontiste une alternative thérapeutique fiable et efficace à certaines malocclusions complexes à traiter avec un dispositif multibague classique.

Nous présentons les techniques chirurgicales de mise en place de ces plaques, leurs indications précises et discuterons des avantages et inconvénients en comparaison aux mini-vis. 